\title{
Joseph Beuys: una visión de las prácticas artísticas contemporáneas y sus posibilidades políticas
}

\section{Artículo de reflexión}

\section{Marcela Quiroga Garza}

Universidad Autónoma de Nuevo León, México marcelaquirogagarza@gmail.com

Recibido: 31 de octubre de 2017

Aprobado: 6 de diciembre de 2017

Como citar este artículo: Quiroga Garza, Marcela (2018). Joseph Beuys: una visión de las prácticas artísticas contemporáneas y sus posibilidades políticas. Calle14: revista de investigación en el campo del arte 13 (24) pp. 332-342. DOI: https://doi.org/10.14483/21450706. 13529 
- 
Joseph Beuys: una visión de las prácticas artísticas contemporáneas y sus posibilidades políticas

\title{
Resumen
}

Hay una relación entre las ideas del artista Joseph Beuys con algunas prácticas y teorías artísticas contemporáneas, mayormente las que se relacionan con acciones directas, cierto activismo y algunos proyectos de desarrollo cultural. Beuys, inserto en un movimiento mundial amplio en los años sesenta, hizo un diagnóstico de las prácticas e instituciones humanas tales como el trabajo, el arte, las relaciones interpersonales, entre otras, y propuso un proceso para restaurar valores perdidos por la racionalización del pensamiento y lo que devino de ello. Este proceso incluye lo que podría ser el remedio del malestar social, el cómo ejecutarlo y su posible resultado. Su legado más importante es constantemente olvidado o dejado de lado por analistas y críticos. Beuys dejó una gran cantidad de páginas de las conferencias que dictó, de las obras procesuales que incluían horas y días de diálogo con grupos de personas y un sinnúmero de entrevistas.

\section{Palabras claves}

Arte contemporáneo, prácticas artísticas, Joseph Beuys, arte políticamente comprometido.

Joseph Beuys: A Vision of Contemporary Artistic Practices and their Political Possibilities

\begin{abstract}
There is a relationship between the ideas of the artist Joseph Beuys and some contemporary artistic practices and theories, mostly those related to direct action, a certain activism and some cultural development projects. Beuys, inserted in a worldwide movement in the sixties, made a diagnosis of human practices and institutions such as work, art, interpersonal relationships, among others, and proposed a process to restore lost values through the rationalization of thought and its consequences. This process includes what could be the remedy of social unrest, how to execute it and its possible outcome. His most important legacy is constantly forgotten or left out by analysts and critics. Beuys left a lot of pages from the lectures that he gave and from his procedural works, including hours and days of dialogue with groups of people and countless interviews.
\end{abstract}

\section{Keywords}

Contemporary art, artistic practices, Joseph Beuys, politically engaged art.

Joseph Beuys : une vision des pratiques artistiques contemporaines et de leurs possibilités politiques

\section{Résumé}

Il y a une relation entre les idées de l'artiste Joseph Beuys et certaines pratiques et théories artistiques contemporaines, principalement liées à des actions directes, à un certain activisme et à des projets de développement culturel. Beuys, inséré dans un mouvement mondial dans les années soixante, a fait un diagnostic des pratiques humaines et des institutions telles que le travail, l'art, les relations interpersonnelles, entre autres, et a proposé un processus pour restaurer les valeurs perdues par la rationalisation de la pensée et ses conséquences. Ce processus comprend ce qui pourrait être le remède de l'agitation sociale, comment l'exécuter et son résultat possible. Son héritage le plus important est constamment oublié ou laissé de côté par les analystes et les critiques. Beuys a laissé beaucoup de pages des conférences qu'il a données, des travaux procéduraux, inclus des heures et des jours de dialogue avec des groupes de personnes et d'innombrables interviews.

\section{Mots clés}

Art contemporain, pratiques artistiques, Joseph Beuys, art engagé politiquement. 
Joseph Beuys: uma visão das práticas artísticas contemporâneas e suas possibilidades políticas

\section{Resumo}

Há uma relação entre as idéias do artista Joseph Beuys com algumas práticas artísticas contemporâneas e teorias, principalmente aquelas relacionadas a ações diretas, certo ativismo e alguns projetos de desenvolvimento cultural. Beuys, inserido em um movimento mundial nos anos 60, fez um diagnóstico das práticas e instituições humanas, como trabalho, arte, relações interpessoais, entre outras, e propôs um processo para restaurar os valores perdidos pela racionalização do pensamento e suas conseqüências. Esse processo inclui o que poderia ser o remédio da inquietação social, como executá-lo e seu possível resultado. Seu legado mais importante é constantemente esquecido ou deixado de lado por analistas e críticos. Beuys deixou um monte de páginas das palestras que deu, dos trabalhos processuais, incluindo horas e dias de diálogo com grupos de pessoas e inúmeras entrevistas.

\section{Palavras chaves}

Arte contemporânea, práticas artísticas, Joseph Beuys, arte politicamente engajada.

\section{Joseph Beuys: kawaspa ruraspa ñugpamanda parlangapa kunaurramandakunata}

\section{Maillallachiska:}

Kaipi kai runa Joseph Beuys suti nikumi nukanchi llullaringa suma kausangapa. Kai iuatikunapi man tianchu respitu kawipi taita i chasallata mama lisinsiarinkuna wambrakuna mandangapa chiminda kunaura kausanchi Imasa munaskasina. Pai niku maskangapa sug mailla ambi mailla mailla aidachingapa parlukunaua allilla kasusangapa

\section{Rimangapa Ministidukuna:}

Ñugpamanda ruraikuna, nukanchi kikinta kilka, Joseph Beuys, runa kasa suti, kawachispa tukuikunata Nukanchipa kagsina. 


\section{Introducción}

En el siguiente ensayo se pretende hacer un análisis de los textos de Joseph Beuys Hablar de mi país y What is art? ${ }^{1}$, así como de algunas otras entrevistas de él publicadas por diversos autores, con la finalidad de observar su vigencia y la influencia que tiene en algunas manifestaciones contemporáneas de arte, activismo y desarrollo de proyectos culturales; y por otro lado, enunciar los elementos por los cuales es pertinente tomarlo como referencia y sustento teórico en diversos proyectos, tanto de creación como de desarrollo cultural.

El preámbulo para contextualizar lo político en las prácticas contemporáneas se desarrollará a partir de los conceptos de Hal Foster. El texto ${ }^{2}$ de Foster al que se hará referencia en este ensayo y que se revisará a la luz de las conferencias de Beuys fue publicado en 1985 un año después de la muerte del artista alemán. La relevancia es observada a partir de la relación que tienen la cultura y la economía en la teoría de Beuys, ambos elementos retomados por Foster para hacer su comentario acerca de las implicaciones políticas del arte contemporáneo.

\section{Foster: el proyecto político del arte actual}

Es necesario hacer una aclaración. En el texto Hablar del propio país: Alemania, Beuys manifiesta reiteradamente que el uso de la palabra 'político' le es imposible (Beuys, 1986) y cuando se refiere a aquello que implica para él este concepto, habla por ejemplo del partido verde alemán. Al parecer, la referencia que hace tiene más relación con la administración de la política (partidos o administración burocrática) que con lo político como una forma de relación entre las personas que comparten determinadas cosas comunes. "Esto quiere decir lo peor y lo más triste de todo, ser capaz de hacer política significa renunciar a todo potencial de ideas futuras" (Beuys, 1986). Sin embargo, lo descrito anteriormente, las definiciones de escultura social producida en procesos dialógicos por medio de la lengua como elemento común, vislumbra una posición clara acerca de la capacidad política del arte.

\footnotetext{
1 Traducido por la autora: ¿Qué es el arte?

2 Recodificaciones: hacia una noción de lo político en el arte contemporáneo fue publicado un año después de la muerte de Beuys en 1985 y retomado por los compiladores del texto Modos de hacer. Arte crítico, esfera pública y acción directa, editado en 2001.
}

Foster propone que la separación marxista de cultura y economía pervivía hasta hace muy poco en los teóricos que se ocupaban de los análisis (menciona a Delphy, Lefebvre y Barthes) y analiza que la realidad actual integra ambos campos:

Y sin embargo esta parece ser la realidad con la que nos enfrentamos hoy día: la ruptura de la antigua oposición estructural entre lo cultural y lo económico debido a la 'comodificación' de la primera y a la 'simbolización' de la última (Foster, 2001b).

Para Foster el carácter político del arte es aquel en el que la capacidad de transformación es un problema nuevo devenido de un escenario anterior planteado por la modernidad, las vanguardias artísticas del siglo $X X$, los movimientos obreros y la lucha de clases. Las vanguardias coadyuvaron a los movimientos obreros en su lucha versus la opresión del capitalismo y la burguesía, tenían un planteamiento hasta cierto punto claro. Benjamin incita a los artistas a cerrar filas con el proletariado en su texto El autor como productor, sin embargo, el proyecto vanguardista falla porque en su afán de emancipar al ser humano no tomó en cuenta que la hegemonía tiene otras formas de sujeción que no son precisamente las que se dan en el nivel laboral. Los movimientos vanguardistas terminan subsumidos por el sistema contra el cuál reaccionaron contestatariamente y terminan siendo su mejor forma de promoción. El poder hegemónico es transparente y tiene en la escena cultural su campo de batalla.

Según dicha lógica, la hegemonía de las representaciones no puede ser contestada únicamente mediante la lucha de clases convencional, puesto que la hegemonía opera a través de la sujeción cultural, tanto o más que mediante la explotación económica (Foster, 2001a). Aquí se encuentra una relación directa con una de las ideas que maneja Beuys en su conferencia Hablar desde el propio país: Alemania. La vanguardia para Foster estuvo al servicio del capitalismo, para Beuys el arte moderno ${ }^{3}$ es una señal que sólo la burguesía puede descifrar $y$, sin embargo, le sirve a él para indicar que algo debe cambiar.

Foster plantea que ante el panorama hegemónico que controla al sujeto por medio de la cultura y ante los embates del capitalismo, más que una batalla de

3 Beuys define vanguardia de la siguiente forma: "es una especie de punta que avanza en otra dirección para escapar de la situación presente" (Rona, 1994). 


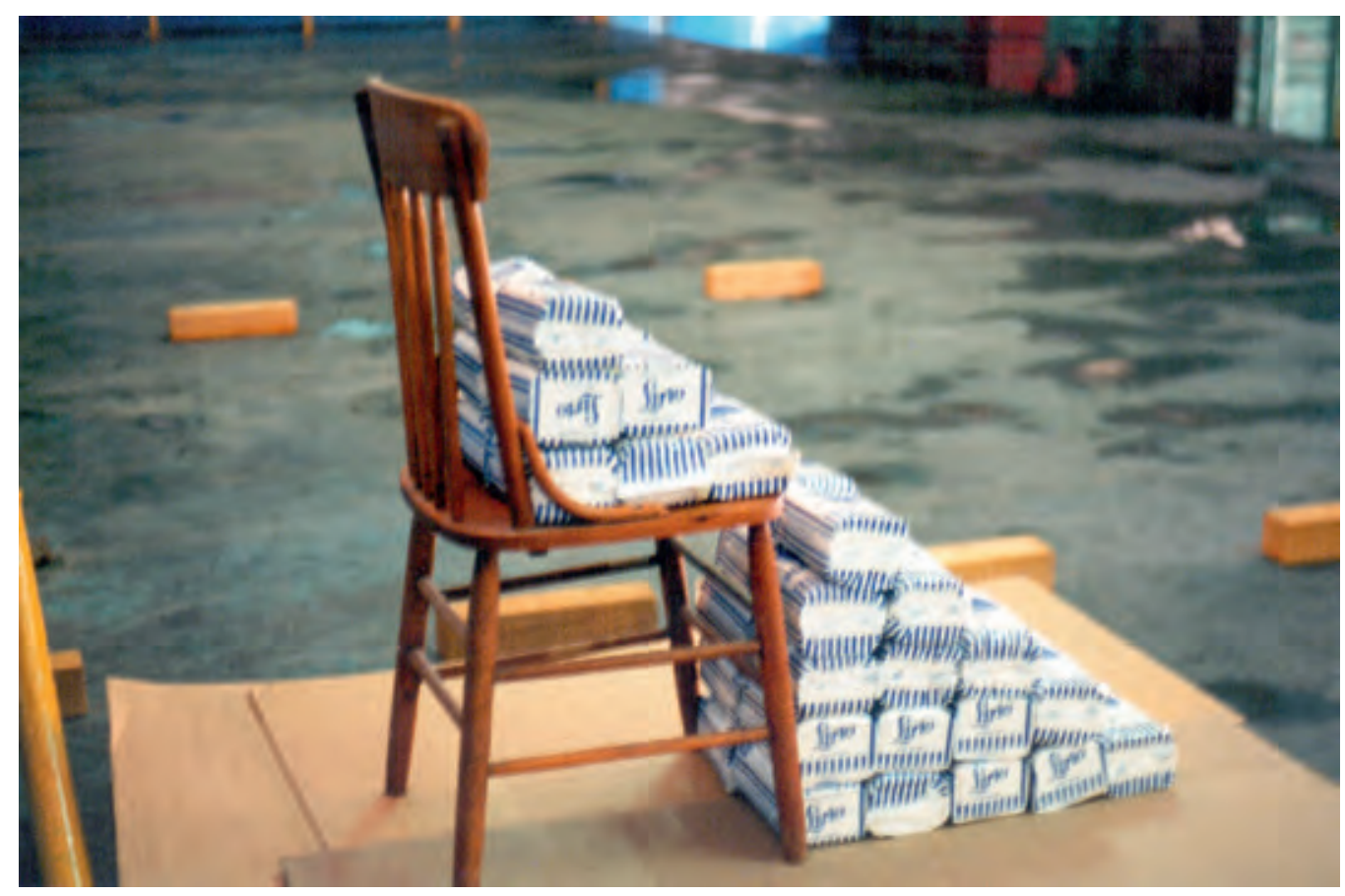

Proyecto: time ES money. Autores: Colectivo CAXA. Instalación en la fábrica de Industrias González, Monterrey, N.L. México. Fecha: agosto 17 de 1996 (Material de registro de autor no identificado).

confrontación como la que libró la vanguardia lo que se debe hacer es resistir, echar en marcha una estrategia deconstructiva, asumiendo un sujeto integrado al sistema. La confrontación frontal implica un afuera natural desde el cual pugnar, pero en la resistencia todo está colonizado: desaparece el afuera, no existe (Foster, 2001b).

Este es otro de los puntos de encuentro con Beuys. La escultura social no exige confrontación, sino la creación de redes interpersonales que cuestionan y debaten las cosas que les atañen por medio de lo que tienen en común: la lengua.

La práctica artística para Foster tendría que dejar de reproducir los aparatos de control porque no extienden el espacio de acción, dejar de representar para constituir un proceso de acción.

Aquí debemos distinguir, por tanto, entre un 'arte político' que, enclaustrado en su código retórico, se limita a reproducir representaciones ideológicas, y un 'arte con política' que, preocupado por el posicionamiento estructural del pensamiento y por la efectividad material de su práctica dentro de la totalidad social, busca producir un concepto de lo político de relevancia para el presente. Indudablemente el acceso a este concepto es difícil y provisional, pero eso mismo puede ser la prueba de su especificidad y la medida de su valor (Foster, 2001b).
En el mismo sentido, la práctica artística en el contexto de la frase de Beuys 'todo ser humano es un artista', desmonta la realidad dada en forma de cuestionamiento que activa acciones desde un plano que le permite crearse a sí mismo no desde la trinchera del arte, sino desde cualquier campo de desarrollo humano. [...] me refería a la capacidad en cada puesto de trabajo, y me refería a la capacidad de una enfermera o la capacidad de un agricultor como potencia conformadora, y a reconocerlas como pertenecientes a un planteamiento de tareas artísticas (Beuys, 1986).

\section{Beuys y las ideas. Diagnóstico: el arte moderno como señal}

En el texto Hablar de mi propio país: Alemania el arte moderno le sirve a Beuys como detonante para hablar del estado en el que, a su parecer, se encuentra no sólo el arte, sino las relaciones entre las personas y el mundo en general. El panorama que plantea Beuys acerca del estado del arte moderno es claro, el arte ya no opera como visibilidad de las cosas comunes de los pueblos ${ }^{4}$.

Con la palabra pueblo hace referencia a la lengua, es decir, al componente básico común compartido entre personas: "El concepto de pueblo está vinculado de manera elemental con su lengua" (Beuys,1986) 


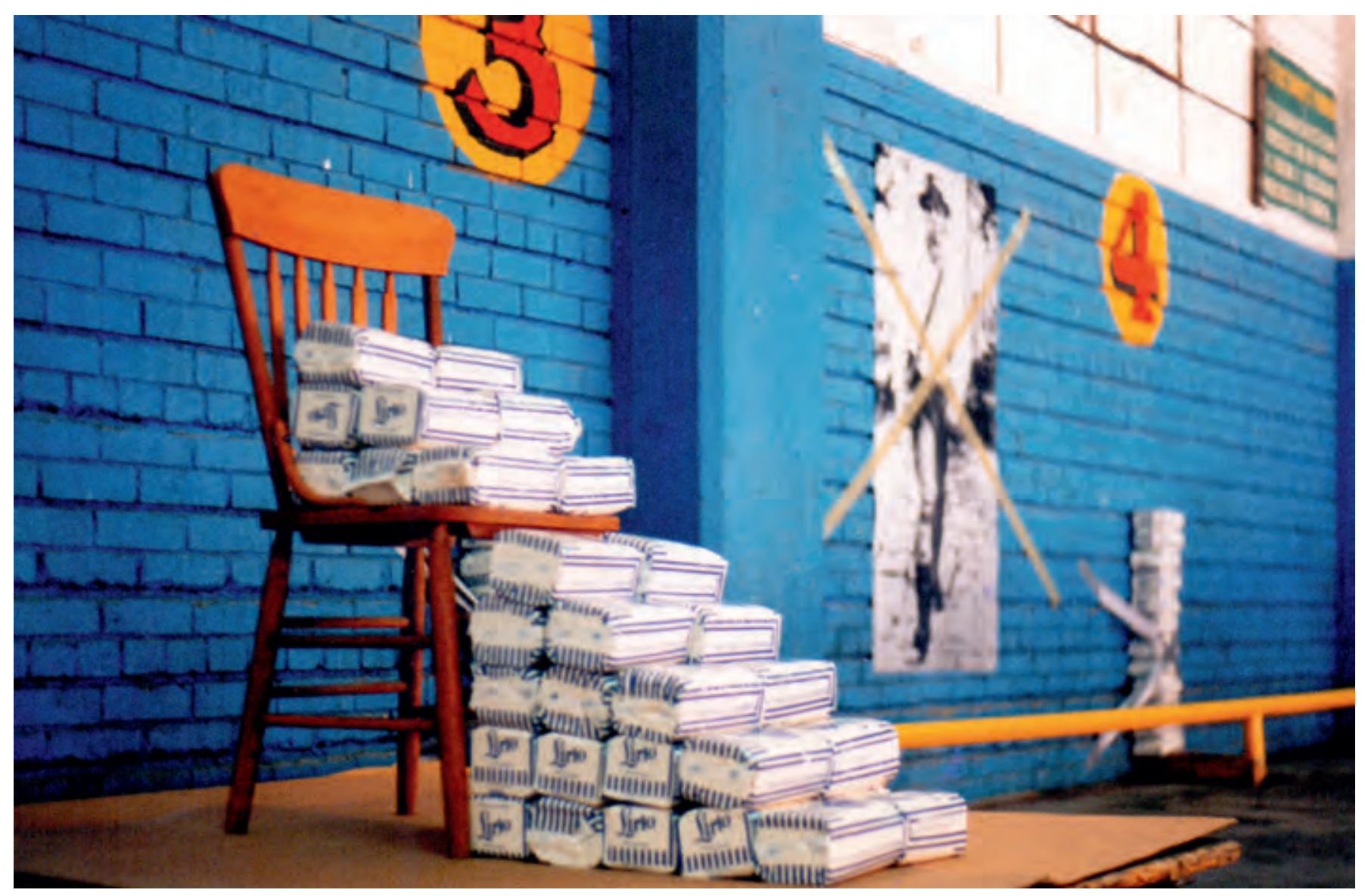

Proyecto: time ES money. Autores: Colectivo CAXA. Instalación en la fábrica de Industrias González, Monterrey, N.L. México. Fecha: agosto 17 de 1996 (Material de registro de autor no identificado).

Yo me percaté de que en mi campo de trabajo, el arte, impera un concepto (o ningún concepto) que ya no es operativo. Este concepto inexistente tiene un carácter afirmativo y afirma de sí mismo lo que no es capaz de producir (Beuys, 1986).

Beuys observa que la capacidad del arte de contrarrestar los efectos de los sistemas hegemónicos como el capitalismo o el socialismo (aún operante en el tiempo de la conferencia) es nula debido a que los procesos por medio de los cuales las personas tienen acceso a él han ido cerrándose paulatinamente después de la época renacentista.

"I found that art undergone a kind of parallel development with science, an academicism, with a long tradition going back to the Renaissance, and the people no longer knew exactly what they were trying to do" (Beuys, 2004).

El arte moderno para Beuys tiene un carácter de señal que sólo puede ser descifrada por aquella clase social que tuvo acceso al conocimiento del proceso por el cual se llegó a tal estado, es decir, la burguesía. El arte se muestra como un enigma descifrable sólo para algunos y por tanto ajeno para todas las demás personas. "Estos hitos o señales en el arte moderno, tal y como han sido mostrados al hombre como símbolos y enigmas, ... Este gran carácter de señal, que ha dejado solos a la inmensa mayoría de los hombres" (Beuys, 1986).

Beuys apunta que la sintomatología que encuentra en las condiciones del arte moderno no solo es evidencia de su estado, sino también de la condición de las relaciones que las personas tienen entre sí, con otras formas de vida y con el mundo (planeta tierra). El carácter de señal para él tiene relación con esto y es el aviso de que algo debe cambiar y para ello es crucial que la señal sea descifrada por todas las personas. La forma en que la vida se organiza en los sistemas hegemónicos no permite la participación en el arte de la mayoría debido a que están en su puesto de trabajo, por ende, lo que debe cambiar es la forma en que este se estructura.

\section{[...] como no habría podido ser de otro modo, dado} que la inmensa mayoría de los hombres tenía otras 
necesidades y cuidados que los de los artistas con la obra de arte y sus disfrutadores. Para qué era una señal esta trágica situación, tuve que preguntarme, aquí la obra de arte se me tornó un enigma para el que el hombre mismo tenía que ser la solución: la obra de arte es el enigma más grande de todos, pero el hombre es la solución (Beuys, 1986).

Beuys adjudica la matriz de la problemática que encierra el sistema hegemónico al control que el Estado y el capitalismo privado tiene sobre los sistemas de producción. A su parecer, estos han envilecido el alma humana sobre todo por considerar al dinero como un valor capital y por consiguiente al consumo como una forma de vida. Todo este engranaje inicia en las escuelas y universidades, que al ser controladas por intereses ya sea del Estado o del capitalismo privado, educan al ser humano como una pieza más de la maquinaria que hace funcionar al sistema. Todo este mecanismo al igual que el del arte/señal, se cierra en sí mismo de tal forma que se vuelve inentendible, así la administración de la vida no puede estar en manos de las personas sino de terceros con intereses particulares.

Esto es, se educa en los hombres un instinto muy bajo para que crean en el dinero, en el sentido de sus ventajas. Estamos acostumbrados a escuchar que en el terreno de la producción las empresas tienen por supuesto que extraer beneficios. Porque de otro modo no podrían subsistir, según la ideología. Pero eso es una pura insensatez, ya que yo tengo que describir el ente economía como una cosa que no tiene que servir para nada más que para cubrir las necesidades humanas, y eso en todo el planeta (Beuys, 1986).

Para Beuys el arte debe recuperar la función que perdió en el transcurrir de la modernidad y que arrastró consigo la visión integral de la humanidad por la racionalización del pensamiento. En este proceso recuperaría la autodeterminación para poder hacerse cargo de la administración de su vida y su libertad.

\section{Campo expandido del arte, creatividad y proceso dialógico: escuelas y universidades liberadas}

Beuys crea el concepto de arte social o arte antropológico para explicitar aquello que él cree que puede contrarrestar el estado, tanto del arte como de la humanidad y el planeta. Si el ser humano es el único que puede resolver el enigma del arte, tendría que ser porque es capaz de crear y su creación más importante sería él mismo. Para ello tendría que crear una estructura que le permitiera este proceso en soltura para poder decidir cómo es que quiere vivir. Para Beuys el propósito final del arte social tiene en la expresión 'todo ser humano es un artista' su concepto cumbre. La mayoría de las veces mal entendida, esta frase encierra lo que para él podría ser un proceso de liberación del arte ahí donde está cautivo, de la humanidad, de la esclavitud moderna y del planeta de la explotación.

En el inicio del discurso Hablar del propio país: Alemania manifiesta -y esto puede ser deducido por el lector-, que al hablar de lengua se refiere al elemento que se comparte con otros, al vehículo de expresión que hace comunidad y es por ello que lo enuncia en términos de originalidad y genio. Propone que el uso de la lengua ${ }^{5}$ nos proporcionaría el espacio para el diálogo. En el ejercicio de hablar se configura el yo consciente, se crean relaciones interpersonales y nuevas formas de nombrar el mundo. La discusión funcionaría como un proceso escultórico social que se constituye en una acción determinada por el lenguaje que da acceso a una realidad común.

El primer eslabón de la cadena de esta escultura social fue planteado por Beuys desde su propia práctica artística, proponiendo que, desde el proceso de pensar y hablar (el proceso que él llevó a cabo), se podría extender no sólo a las demás prácticas artísticas sino a otros campos humanos.

My objects are to be seen as stimulants for the transformation of the idea of sculpture, or art in general. They should provoke thoughts about what sculpture can be and how the concept of sculpting can be extended to the invisible materials used by everyone: Thinking Forms-how we mould our thoughts or Spoken Forms-how we shape our thoughts into words or SOCIAL SCULPTURE how we mould and shape the world in which we live:

5 Beuys habla de la lengua alemana y asumo dos cosas: que la conferencia es dictada en Alemania y que por esto habla en términos locales, lo que comparte en común con sus coterráneos. La anotación tiene como motivo aclarar que los debates en torno al nacionalismo no interesan en este ensayo y serán pasados por alto, así como la influencia que Beuys pudiera tener de la escuela naturalista alemana. 
Sculpture as an evolutionary process; everyone an $\operatorname{artist}^{6}$ (Beuys, 2004).

En este sentido la concepción del arte se expande hacia la esfera antropológica, la esfera de la realidad humana. Para Beuys, por medio de la escultura social se superaría la crisis profunda de la humanidad porque el ser humano se determinaría a sí mismo y administraría su vida y el cambio en la estructura del sistema se activaría.

Esta circunstancia debe aportarnos una posibilidad real de cambiar el sistema social. En razón de este concepto de arte antropológico, cada hombre es un artista. En cada hombre existe una facultad creadora virtual. Esto no quiere decir que cada hombre sea un pintor o un escultor sino que existe una creatividad latente en todas las esferas del trabajo humano (Rona, 1994).

Para Beuys cambiar el sistema social partiendo del concepto expandido del arte es una posibilidad derivada de la capacidad creativa que daría un giro al engranaje del sistema social, sus mecanismos, su motor y su visibilidad.

Hablo de la creatividad en todos los hombres y no solo en algunos creadores. Hablo de la creatividad en todas las actividades y en todas las formas de trabajo, no solo en el arte; de una creatividad que libere al trabajo y lo eleve a la categoría de acto libre y revolucionario (Rona, 1994).

En este proceso ningún momento de la vida desde el nacimiento hasta la muerte está fuera. Cada experiencia, cada palabra, cada imagen constituyen un recurso en flujo que se constituye desde la vida cotidiana en el cuestionamiento constante que provoca y estimula la discusión.

"In other words, I have to keep preparing myself throughout my life, conducting myself in such way that no single moment is not given over to this preparation. [...], I must always have the presence of mind, the

6 Traducido por la autora: Mis objetos deben ser vistos como estimulantes para la transformación de la idea de la escultura, o el arte en general. Ellos deben provocar pensamientos sobre lo que la escultura puede ser y cómo el concepto de la escultura puede extenderse a los materiales invisibles utilizados por todos: formas de pensamiento —cómo moldeamos nuestros pensamientos o formas hablada - cómo formamos nuestros pensamientos en palabras o ESCULTURA SOCIAL cómo Moldear y moldear el mundo en el que vivimos: Escultura como proceso evolutivo; todo el mundo un artista (Beuys, 2004). overview, the wider perspective, to perceive the overall context and set of forces"7 (Beuys, 2004).

Las instituciones culturales primordiales son la escuela y las universidades. Al ser controladas por el Estado operan como un mecanismo del mismo y en función de sus intereses. Para Beuys estas deben ser liberadas y auto-administradas. Desde el campo expandido del arte estas instituciones funcionarían como productoras del capital creativo que en el sistema renovado es el valor económico.

El valor económico es la capacidad humana empleada en el trabajo, y lo que surge como producto en el puesto de trabajo, una buena escultura, un cuadro maravilloso, un auto que no contamina el medio ambiente, una patata sabrosa y sana, un pescado sano, ... (Beuys, 2004).

\section{Seres auto-determinados, instituciones auto-administradas}

El arte, planteado como un proceso en constante construcción desde el que Beuys proyecta una posibilidad de renovación social, puede guiar un desarrollo holístico del mundo. Al igual que el campo del arte se expande hacia un proceso de escultura social por medio del pensar y el hablar, en esta visión holística el contexto también se expande: "This is the bigger context, in the scheme of which all possible forms exist ${ }^{8 "}$ (Beuys, 2004). Desde aquí, el ser humano que vive en esta forma de organización nueva, auto-determina por medio de su voluntad su forma de vivir y su experiencia. Es el creador de su vida y del mundo que también construye:

"The human being himself becomes creator of the world and experiences how he can continue creation. This now also becomes his responsibility, and all the facts and realities connected with this become visible"9 (Beuys, 2004).

\footnotetext{
7 Traducido por la autora: En otras palabras, tengo que seguir preparándome a lo largo de mi vida, conduciéndome de tal manera que ningún momento no sea sino para esta preparación. [...], siempre debo tener la presencia de la mente, el panorama, la perspectiva más amplia, para percibir el contexto general y el conjunto de fuerzas (Beuys, 2004).

8 Traducido por la autora: Este es el contexto más grande, en cuyo esquema todas las formas posibles existen (Beuys, 2004). $9 \quad$ El ser humano se convierte en el creador del mundo y las experiencias para continuar esta creación. Esto se convierte en su responsabilidad, y todos los hechos y realidades relacionados con esto se vuelven visibles (Beuys, 2004).
} 


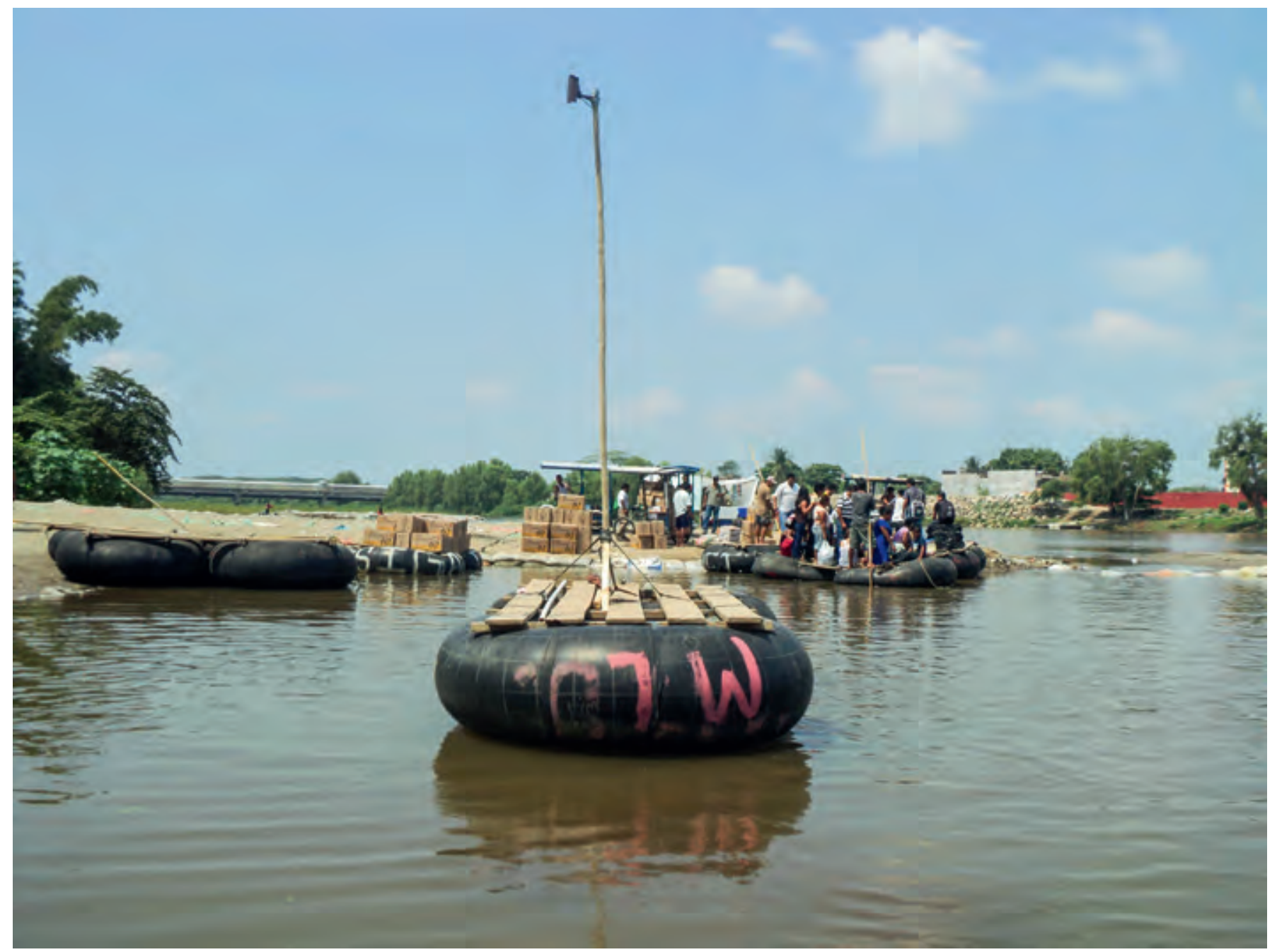

Proyecto: ¿Cuál realidad? Cámara que vigila al cielo. Autora: Marcela Quiroga. Instalación de cámara de vigilancia montada en balsa utilizada en el punto fronterizo alterno de México con Guatemala en el Río Suchiate entre las ciudades de Cd. Hidalgo y Tecún Unam. 2010-2011.

La auto-determinación trae como consecuencia la auto-administración de la forma en la que se produce la vida. Las instituciones culturales, escuelas y universidades, su trabajo y la forma en la que se distribuye, serían gestionadas con y por otros intereses, sin el factor condicionante del dinero que en el ciclo capitalista nos obliga a ser consumidores de cosas. Esto para Beuys es lo que ancla al ser humano a una existencia materialista: el ser humano es esclavo de los objetos que puede comprar.

Ya puede percibirse y observarse que el hombre, con creciente exigencia y con el surgimiento de su autoconciencia y de su libertad, se suelta poco a poco los grilletes de los egoísmos materiales. Cierto es que el egoísmo pervive en nosotros con mucha fuerza, pero sin embargo vemos, sobre todo si nos dedicamos a esta tarea con ejercitación creciente, un progresivo liberarse de los vínculos. El ego, el yo, exige un alimento espiritual a través de la economía. Este yo que exige un alimento espiritual se percata también de que la rama más importante de la economía radica en el ámbito de la producción, digo en el ámbito de la producción y no en el ámbito del consumo, allí donde se trabaja de acuerdo con el principio de la división del trabajo, no donde actúan la pequeña economía y las profesiones libres, sino que me refiero conscientemente a las empresas más importantes en el terreno de la producción, las escuelas y los centros de enseñanza superior. En ellos se produce el CAPITAL de la sociedad. Repito CAPITAL no es dinero (medio de producción), CAPITAL es capacidad y producto de la capacidad (Beuys, 1986).

En estos enunciados Beuys plantea que la producción en este modelo nuevo sería el resultado de un proceso de significación del mundo a partir de un proceso creativo. La economía estaría basada en la cultura lejos del intercambio monetario (pesos, dólares, yenes, otros.) que rige nuestro sistema. El dinero no condicionaría la producción y con ello tampoco la vida. 

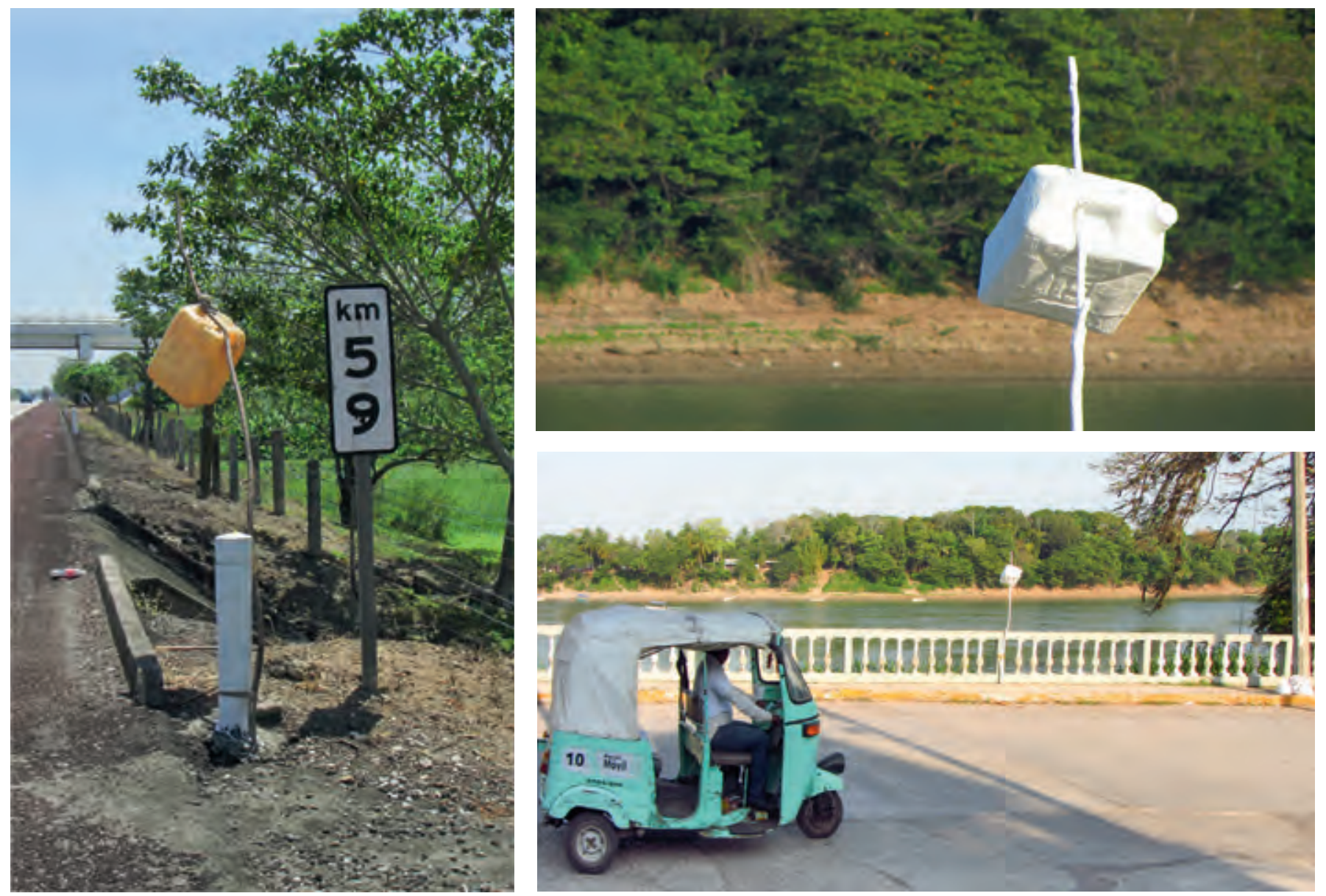

Proyecto: ¿Cuál realidad? Tanque en palo. Autora: Marcela Quiroga. Fotografías documentales y de registro del contrabando y venta de gasolina en la frontera entre Tenosique México, con Guatemala. Instalación de escultura en sitio.

\section{Referencias}

Beuys, J. (1994) Catálogo de la exposición. Madrid: Museo de Arte Reina Sofía.

(1986) "Hablar del propio país Alemania". En: En torno a la muerte de Joseph Beuys, Necrologías, ensayos, discursos. Bonn: Inter Nations, pp. 37-58. Impreso.

(2004) What is art? Conversation with Joseph Beuys. Edited by Volker H. Trad: Matthew Barton and Shelley Sacks. United Kingdom: Claireview.

Bernárdez, C. (1999) Joseph Beuys. Madrid: Nerea.
Foster, H. (2001a) El retorno a lo real, la vanguardia a finales de siglo. Trad: Alfredo Brotons Muñoz. Madrid: Akal.

(2001b) "Recodificaciones: hacia una noción de lo político". en: Modos de hacer: arte crítico, esfera pública y acción directa. Salamanca: Ediciones Universidad de Salamanca.

Rona, E. (1994). Entrevista con Joseph Beuys. en: Lamarche-Vadel, Bernard, Joseph Beuys. España: Siruela. 